\title{
At the far end of diachrony: the evolutive spiral of the emergence of language. A theory
}

\author{
Adina Chirilăa \\ Faculty of Letters, History and Theology, West University, Bd. Vasile Pârvan 4, 300223 Timişoara, Romania
}

\section{Article info}

History:

Received October 24, 2019

Published November 7, 2019

Key words:

neurolinguistics

mirror neurons

mirror system hypothesis

origin of languages

\begin{abstract}
Among the analytical efforts that pursue the question of the emergence of language, and possibly the formation of the cerebral mechanism that made it possible, the hypothesis of the mirror system developed by Michael A. Arbib is a well-articulated one, due to an interdisciplinary approach. The present article highlights several crucial elements in the process of its elaboration and exposes its essence: 1 . there is no innate universal grammar; 2 . language-readiness is a multimodal state - it evolved as a multimodal system: manual/facial/vocal, by stages: a) first, the protosign (manual protolanguage, with an open repertoire of signs); b) then, the protospeech (vocal protolanguage), that provided "the critical neuronal mass" due to which c) language evolved from protolanguage, as a result of cultural innovations; 3 . the brain mechanisms that sustain language evolved atop a primitive pre-hominid mechanism that initially had nothing to do with communication; this, i.e. the mirror system for grasping, provided the evolutive base for language parity.
\end{abstract}

Prologue. A considerable number of articles and books-be they addressed to a highly specialized public, or to the common reader with a decent interest in sciences-whose object are the so-called mirror neurons (or which only rely on what it is known about them in order to explain this or that behavioural phenomenon) reserve at least one paragraph for summarising the history of their discovery in the frontal cortex of the macaque monkeys (the ventral premotor region, called F5, then in the inferior parietal lobule-including the ventral and lateral intraparietal areas, as well as in the dorsal premotor cortex and the prime motor cortex) twenty seven years ago (Di Pellegrino et al., 1992; see also Rizzolatti et al., 1996; Gallese et al., 1996; Fogassi et al., 2005, etc.). Through such papers and books, people have quickly understood that mirror neurons are visuomotor neurons that react at the observation of the motor acts executed by a different individual, their special property being that of creating a certain identity between the $o b$ servation of manual or oral movements and the execution of identical or similar movements (Rizzolatti \& Fogassi, 2014); in simpler words, the neuronal system in question makes use of a single neural code for two rational capacities: observation and execution of an action. What follows is that the mechanism provides the observer with the automatic understanding - i.e. a comprehension without inferential processing - of the motor act of the observed individual (Rizzolatti \& Fogassi, 2014), a connection between the observer and the observed being thus established.

When and to what extent it was possible, the researches conducted on human subjects (Molenberghs et al., 2013; Mukamel et al., 2010; Kilner et al., 2009; Chong et al., 2008; Press et al., 2012) confirmed the existence of a wide correspondence between the cortical areas active in observation-execution in humans, and the areas in which mirror-neurons had been detected in macaques; this discovery raised the hypothesis that a system of mirror neurons might be present and functional in the human brain as well (Kilner et al., 2013, p. 1060-1061).

\footnotetext{
^Email address: chiriladina@yahoo.com.
} 
The effect of the constant reiteration of these mirror neurons' mechanism in a manner rather simplified, that was obliterating many aspects still unclear $^{1}$, was that in the mind of the cultivated yet common reader a bright and powerful idea flourished: namely, that this class of visuomotor neurons is the corner stone of more or less vaguely expressed edifices, from social cognition, and "the great leap forward" (Heyes, 2010) in the evolution of humans, to a plethora of abilities, disorders and phenomena, like speech, schizophrenia, hypnosis, perception (sometimes painful) of amputated limbs, psychopathy, smoking, love, empathy, autism, paranoid hallucinations, political attitudes.

It seemed that everything and anything could be explained through the actions of the mirror neurons, although indeed only a fraction of the researches had accomplished the task of irrefutably (or at least plausibly) demonstrating their hypotheses, following a methodology that would have not given a chance to conclusions that, although enthusiastic and seductive to the public, were in fact pure speculative. Nevertheless, at some point, this seeming theoretical all-inclusiveness has driven the explanation to collapse under its own popularity; the suspicion one might have had, legitimately, concerning the function of the mirror system in a certain phenomenon has extended without discernment upon its function in many (if not all) others. Thus, as in too many cases, the burden of sifting the chaff from the wheat devolves upon the reader-a task as difficult when everything is advertised as wheat as when everything is advertised as chaff.

The Theme. Among the phenomena or abilities related to human beings, the language is one of the firsts that people tried to explain through some property of the mirror neurons.

Science provides many a scenario about the emergence of language ${ }^{2}$, complementary or assumed antagonistic ${ }^{3}$, in which the scientists (from domains remote from the traditional science of language - e.g. biology, neurology, computational sciences, engineering-, and more and more eclectic) struggle in order to descend one more step towards the "moment" when the language came as an efficient communication tool of the individuals that formed a community. The general impression, however, on the common reader is that of an annoying incapacity to reach beyond a conjectural sketching of some possible/probable social circumstances that might have stimulated the language phenomenon-a necessity imposed by the environment, be it economic and/or political, cultural, etc. But why and how was it possible for the requests of the environment to have the chance of being "listened to", with the result, among many others, of concocting a communication system through what we've come to label as linguistic signs remained, until recently, an unexplained (or, at best, unconvincingly explained) problem. So that, at the very proximate end of the history of language, linguists seem to be right in accepting the easiest solution to the problem: ignoring it.

The idea emanated from the discovery that the mirror system region in the human brain, corresponding to the F5 zone in the macaques' brain, includes the so-called Broca's area, traditionally associated with speech.

It seemed that to establish a connection between (a) mirror neurons, (b) imitation (simple $\rightarrow$ complex), and (c) the emergence/evolution of language could have been a sane approach, that would have

\footnotetext{
${ }^{1}$ See Kilner et al. (2013) for a succinct presentation of the certainties obtained from thorough researches, of the still (in some cases, to this day!) unresolved difficulties, and of the risks that scientists face when they apply the mirror neurons hypothesis indiscriminately, lacking a deep understanding of the phenomena involved.

${ }^{2}$ E.g. "the exchange of information and cooperation", "the consolidation of social relations", "technological pedagogy", "the negotiation", "the need to dominate rival groups", "social manipulation", "the specificity of the (political) niche in which homo got to live", "the narration of real or imaginary events", "the necessity to clarify one's own ideas", "language - consequence of intelligence"...

${ }^{3}$ In fact, the rivalry is gratuitous: in many of these theories, the driving force behind the emergence of language is essentially the same and relates to the individuals' response to the environment's demands; "cooperation" (in order to obtain food or safety), "negotiation", "socializing", etc. are different manifestations of the same phenomenon.
} 
explained not only the existence of this particular type of communication capacity among humans, but the very existence of a brain whose mechanisms determined the existence of language-the language ready brain. This approach gives credit to the neurolinguistic perspective that follows the cerebral mechanisms involved in the perception and production of language (Arbib, 2012, p. 48). The concept of a language ready brain (i.e. an innate ability to learn the grammar of a/several language/s) ought to be kept apart from the concept of the universal grammar that in N. Chomsky's (dangerously teleological) theory (Chomsky, 1965, 1980), represents an universal computational structure which functions as archetype-grammar, fixed in the human genome (a biological property, genetic treat), that is able to specialized itself in order to become/to produce the individual grammars of languages ${ }^{4}$.

The Hypothesis. The mirror system hypothesis represents the two decades elaboration of the idea that the linguistic mechanisms appeared and evolved atop the mirror neurons system employed during grasping, grafting on it, profiting from its abilities, and "learning" from it. The artisans of the hypothesis have found the idea that the neuronal system that makes possible the overlapping between observation and execution "provides a necessary bridge from 'doing' to 'communicating', as the link between actor and observer becomes a link between the sender and the receiver of each message" (Rizzolatti \& Arbib, 1998, p. 188) to be plausible and provable. The findings and conclusions of the following years of research have been presented in Arbib (2012)5.

There are three crucial hypotheses developed in the book (p. 160-162), that intertwine: 1. there is no innate universal grammar; 2 . language-readiness is a multimodal state-it evolved as a multimodal system: manual/facial/vocal, by stages: a) first, the protosign (manual protolanguage, with an open repertoire of signs); b) then, the protospeech (vocal protolanguage), that provided "the critical neuronal mass" due to which c) language evolved from protolanguage, as a result of cultural innovations; 3 . the brain mechanisms that sustain language evolved atop a primitive pre-hominid mechanism that initially had nothing to do with communication; this, i.e. the mirror system for grasping, provided the evolutive base for language parity - the property that makes a certain symbolic utterance to have the same meaning for the receptor, and for the speaker.

The Solution. Arbib (2012) suggests that there are several stages in the process that led to the emergence of speech-among which he ponders upon six, during three eras:

\section{"Pre-Hominid}

1. A mirror system for grasping: matching action observation and execution for grasping. Shared with common ancestor of human and monkey

2. A simple imitation system for grasping. Shared with common ancestor of human and great apes

\footnotetext{
${ }^{4}$ Although the theory was designed with the English language in mind!

For a brief and efficient survey of the arguments against Chomsky's concept of universal grammar (adopted, in various forms, by more or less charismatic zealots), see Christiansen \& Chater (2008 - with the subsequent comments of a number of researchers, and the authors' response). Still robust are the quite early interventions of Tomasello (1995; see also Tomasello et al., 1993) against the generative grammar and linguistic nativism postulated by Chomsky, and the language instinct of Steven Pinker - the latter deserving a critique only as far as it identifies with the theory of an universal grammar; otherwise, judging by the denotative value of its name, the hypothesis of a language instinct is null from the beginning, since it contravenes the operational definition of instinct: "a behavioral competency, or set of behavioral competencies, that: (a) is relatively stereotyped in its behavioral expression, and (b) would appear in ontogeny even if an individual were raised in isolation from its speciestypical set of experiences" (Tomasello, 1995, p. 132-133).

${ }^{5}$ The book may be advertised as addressing to the general public, since it offers access to a knowledge that has been gained through the (re)interpretation of findings from fields of maximum specialization; however, it solicits more than some rudimentary notions of biology, evolutionism, ethology, human paleontology, neurology, prehistoric archaeology, comparative primatology, and also the willingness to forgo the traditional and thus comfortable ways of scanning the reality, ways that entitle us to reiterate, as surprising discoveries, observations that are, in fact, obvious for everybody.
} 


\section{Hominid Evolution}

3. A complex imitation system: complex imitation combines the ability to recognize another's performance as a set of familiar movements with the ability to use this recognition to repeat the performance, and (more generally) to recognize that another's performance combines actions that can be imitated at least crudely by variants of actions already in the repertoire, with increasing practice yielding increasing skills

4. Protosign: a manual-based communication system, breaking through the fixed repertoire of primate vocalizations to yield an open repertoire

5. Protospeech and multimodal protolanguage: this rests on the "invasion" of the vocal apparatus by collaterals from communication system based on F5/Broca's area

\section{Cultural Evolution in Homo Sapiens}

6. Language: the transition from action-object frames to verb-argument structures which express them; syntax and a compositional semantics: Co-evolution of cognitive \& linguistic complexity"

(Arbib, 2012, p. 174)

Nevertheless, what he proposes, somehow counterintuitively, is not a linear stretch of events and stages that culminate with the formation of language, in the sense of a gradual transformation of one into another. The stages postulated by, and in the mirror system hypothesis are multidimensional network developments of some mechanisms that, while growing refined and moving away from the stem/stems that generated them, allow their original support to evolve as well (performing its functions and responding to specific needs and pressures coming from the environment), contribute to the strengthening of it, and benefit "at a distance" from the optimisations it has gained meanwhile. Thus, the complex imitation system once established-i.e. a) the ability to analyse and to recognise in someone's behaviour a set of familiar gestures executed in order to accomplish some intermediate goals, b) the ability to imitate, with certain plasticity, the observed behaviour, and c) the ability to understand that someone introduces in their behaviour new gestures, that can be imitated more or less accurately by employing variants of what already exists in the repertoire of the observer (see supra; and also p. 188, 214, 263) ${ }^{6}$-, the evolutive retort has took the form of the emergence of the capacity of pantomime, whose essence consists of the intention to awaken in the mind of an observer the thought of a certain object or event. As opposed to the simple imitation, in which the flux of information is unidirectional, ceasing with the imitator who only observes and reproduces-one might say, for themselves-, the pantomime has a communicative nature: the one who pantomimes receives the information from somewhere and executes the gestures that allude to that information, and he/she does so in order to be observed and to get a reaction from somebody. It is possible to consider now the existence of a protolanguage whose matter is the manual gesture (i.e. protosign) - a structure upon which rests and evolves a protolanguage whose matter is the vocal gesture (i.e. protospeech). The first Homo sapiens comes to possess two communication modes not by discovering in himself, through learning, of some hidden/latent capacity of their brain; but by the fact that their brain creates for itself, physically (i.e. biological evolution, that includes the creation of a genetic foundation favourable to language), this capacity:

"the evolution of a system for voluntary control of intended gestural communication based in part on F5/Broca's area provided the basis for the evolution of creatures with more and more prominent connections from F5/Broca's area to the vocal apparatus. This in turn could provide conditions that led to a period of coevolution of the vocal apparatus and its integration with the neural circuitry for control of gesture and protosign."

(Arbib, 2012, p. 231)

\footnotetext{
${ }^{6}$ All of these implying the fact that "mirror neurons are not restricted to recognition of an innate set of actions but can be recruited to recognize and encode an expanding repertoire of novel actions” (Arbib, 2012, p. 131).
} 
Arbib makes use of a concept that is not new in the theories of evolution ${ }^{7}$, that of the expanding spiral; it means that the two primitive modalities of communication do not manifest themselves in a pure consecutiveness: rather, they stimulate and shape each other, and they both constitute an advantage in the natural selection; thus the protosign reaches the state of a full language alongside the apparition of the first forms of protospeech (p. 178, 261). In the light of what happens later, in phylogeny, the brain that possesses the capacity of generating and coordinating conventional manual, facial and vocal gestures with the view of communicating - with an open repertoire, that exceeds the genetic datum (with instinctual "gestures" that fit specific "situations") — is a language ready brain, although it still lacks syntax, complex semantics and expressivity, and the conceptual structures that lay at the foundation of a modern Homo sapiens' language.

The theory had been previously formulated by Arbib (2005; cf. Wray, 1998, 2002), and it had to face critics (e.g. Tallerman, 2007) that alluded to the insufficiency-in terms of survival of the species and of its advantage over a rival one- of a "partial grammar", and, consequently, to the inconsistency of the theory. No matter how well constructed from an analytical-synchronic perspective (i.e. modern), such objections hide a weak point inside their structure: the inexact evaluation of the circumstances in which primitive communication took place (the cognitive capacities of the species, its interests, the prominence of certain elements of the situational field, and not of others that would appeal to the modern conscience, etc.). The theoretical anachronism is sanctionable:

"One must not think of protohumans as having modern thoughts and just lacking the ability to express them. Protohumans would not have the idea of trying to pantomime the equivalent of arbitrary complex sentences. Rather, they would have had a small stock of protowords that would increase over the generations as pantomimes and vocal performances became conventionalized for situations of sufficient importance or frequency."

(Arbib, 2012, p. 259)

Passing from a paradigm of communication based on gesture and rudimentary naming (the alingual state) to one based on structures more or less complex, with verb and argument structures is the result of cultural evolution (essentially, epigenetic ${ }^{8}$ ) - made possible by the biological evolution of the species-, understood as a series of "processes that shape facets of a human culture in ways that are relatively independent of human biology" (p. viii). Again, the process may be described more accurately as an evolutive spiral, in which the lexicon and the syntax of a language (in fact, of languages used by different groups of Homo sapiens $^{9}$ ) do not represent separated stages of development, but emerge and evolve simultaneously.

The hypothesis of the mirror system as the original foundation for modeling a brain that is apt (ready) for language, through complex imitation (an analytical, multimodal and cumulative mechanism; see supra) finds a strong supportive argument in what one can observe regarding the emergence and the evolution of certain "gestural languages"- systems of gestural communication particular to the communities whose individuals lack the capacities to speak and hear ${ }^{10}$. The geneses and evolutions of such languages

\footnotetext{
${ }^{7}$ And in science, in general (see Gafton \& Gafton, 2017), where, however, 'coevolution' is preferred (see the above-cited fragment).

${ }^{8}$ In the evolutionistic theories of the day, those who turn to Darwinism by rejecting the Neo-Darwinists' reductionist solution, and reinclude Lamarck's views in the equation are developing a multi-dimensional vision of the evolution (see Jablonka \& Lamb, 2005). Although the identity between epigenetic evolution and cultural evolution (discussed as symbolical and behavioural evolution) is not explicitly claimed, the amount of observations of empirical nature, and of facts concerning all species should call for it.

On the matter of the cultural factor in the evolution of language, see Dediu et al. (2013).

9 "It may never be possible to decide on linguistic grounds whether language developed just once (monogenesis) or separately in two or more places (polygenesis). And it will certainly never be possible for linguists to recover the structure of 'protoworld' if indeed monogenesis holds. Nonetheless, it seems more likely that multiple protolanguages developed well before there were languages with anything like the richness of modern human languages" (Arbib, 2012, p. 332).

${ }^{10}$ Namely, the Nicaraguan Sign Language, and the Al-Sayyd Bedouin Sign Language from Negev, Israel, which emerged
} 
vary to some degree, yet different stages in their histories may be regarded as enactments of the processes of observation and analyzation of gestures that have a communicative potential, and of construction of new gestures using elements "recuperated" from the deconstruction of the original ones, and "redistributed". Moreover, the syntax of such languages does not imitate the syntax of their spoken variants; it represents a mirror of the structure and of the circumstances of the communicated action or object.

Although, in the case of language, the ontogeny does not recapitulate the phylogeny, the acquisition of a language by a child or the creation of a completely new one, today, happens due to the same brain mechanisms, biologically moulded, that made possible the emergence of language and its cultural shaping. Or,

„[i]f a child sees a complicated sign, he or she will successfully mimic one or two features at first. I would thus suggest that what might look like breaking complex verb expressions down into sequential morphemes may be a matter of motor simplification rather than a linguistic reanalysis. Breaking complex skills into pieces and then learning how to gracefully reconstitute them is a general property of motor learning, and it should not be counted as a design feature specific to language"

(Arbib, 2012, p. 319)

EPILOGUE. As in the case of any truth that cannot be probated by observation on the spot (or... on the time, in the present case), the pursuit of the issue concerning the emergence of language, and possibly before that, of the formation of the cerebral mechanism that made the language possible exposes the researcher to the risk of not sounding anything but a speculative theory. Elegant (in mathematical terms ${ }^{11}$ ), mesmerising perhaps, consistent and coherent within the frames it has established for itself, yet vulnerable when facing a different thinking paradigm that operates with precise details from various fields of studydetails and evidences that must harmonize with each other, that are relevant in fact to the issue in question, and that should have been taken into consideration from the beginning. Michael A. Arbib's Mirror System Hypothesis is, still, the only hypothesis that avoids a risk as such by letting itself be controlled and shaped by a vigorous interdisciplinarity that knows and defends, in accordance with each and every domain, its individual certitudes.

Lately, the research conducted upon various aspects of the same issue ${ }^{12}$ has offered more and more analytical and empirical proofs that reaffirm the robustness (in systemic terms) of Michael A. Arbib's hypothesis.

\section{Bibliografie}

Arbib, M.A. (2005). From monkey-like action recognition to human language: An evolutionary framework for neurolinguistics, in "Behavioral and Brain Sciences", 28 (2), p. 105-124, Crossref.

Arbib, M.A. (2012). How the Brain Got Language. The Mirror System Hypothesis, Oxford University Press, Crossref.

Arbib, M.A. (ed.) (2018). How the Brain Got Language. Towards a New Road Map, special issue of "Interaction Studies. Social Behaviour and Communication in Biological and Artificial Systems", 19 (1-2), Crossref.

Chomsky, N. (1965). Aspects of the theory of syntax, MIT Press, Crossref.

Chomsky, N. (1980). Rules and representations, Columbia University Press / Blackwell.

Chong, T.T., Cunnington, R., Williams, M.A., Kanwisher, N. \& Mattingley, J.B. (2008). fMRI adaptation reveals mirror neurons in human inferior parietal cortex, in "Current Biology”, 18 (20), p. 1576-1580, Crossref.

Christiansen, M.H. \& Chater, N. (2008). Language as Shaped by the Brain, in “Behavioral and Brain Sciences”, 31, p. 489-558, Crossref.

and developed spontaneously, in relatively closed communities, independently of other similar systems with a "grammar" and a "lexicon"

${ }^{11}$ One might find this quality in Chomskyanism; see Tomasello (1995, p. 136).

${ }^{12}$ See, for instance, the articles published in Arbib (2018); also, Corballis $(2015,2017,2019)$. 
Corballis, M.C. (2015). What's left in language? Beyond the classical model, in "Annals of the New York Academy of Science", 1359 (1), p. 1-16, Crossref.

Corballis, M.C. (2017). The evolution of lateralized brain circuits, in „Frontiers in Psychology”, 8, Crossref.

Corballis, M.C. (2019). Minimalism and Evolution, in "Frontiers in Communication", 4, Crossref.

Dediu, D. et al. (2013). Cultural Evolution of Language, in Richerson, P.J. \& Christiansen, M.H. (eds), Cultural Evolution: Society, Technology, Language, and Religion, MIT Press, p. 303-332.

Di Pellegrino, G., Fadiga, L., Fogassi, L., Gallese, V. \& Rizzolatti, G. (1992). Understanding motor events: a neurophysiological study, in "Experimental Brain Research", 91 (1), p. 176-180, Crossref.

Fogassi, L., Ferrari, P.F., Gesierich, B., Rozzi, S., Chersi, F. \& Rizzolatti, G. (2005). Parietal lobe: from action organization to intention understanding, in "Science", 308, p. 662-667, Crossref.

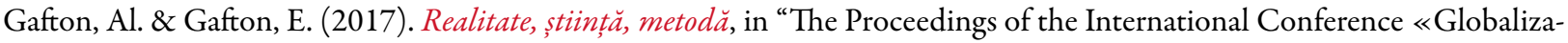
tion and intercultural dialogue: multidisciplinary perspectives»”, 4, Arhipelag XXI Press, Tîrgu Mureş, p. 9-24.

Gallese, V., Fadiga, L., Fogassi, L. \& Rizzolatti, G. (1996). Action recognition in the premotor cortex, in "Brain”, 119 (2), p. $593-$ 609, Crossref.

Heyes, C. (2010). Mesmerising mirror neurons, in "Neuroimage", 51 (2), p. 789-791, Crossref.

Jablonka, E. \& Lamb, M.J. (2005). Evolution in Four Dimensions. Genetic, Epigenetic, Behavioral, and Symbolic Variation in the History of Life, MIT Press (2 $2^{\text {nd }}$ edition, 2015).

Kilner, J., Neal, A., Weiskopf, N., Friston, K.J. \& Frith, C. (2009). Evidence of mirror neurons in human inferior frontal gyrus, in "Journal of Neurosci.", 29 (32), p. 10153-10159, Crossref.

Kilner, J. M. \& Lemon, R.N. (2013). What We Know Currently about Mirror Neurons, in “Current Biology”, 23 (23), p. 10571062, Crossref.

Molenberghs, P., Cunnington, R. \& Mattingley, J.B. (2012). Brain regions with mirror properties: a meta-analysis of 125 human fMRI studies, in "Neuroscience \& Biobehavioral Reviews", 36 (1), p. 341-349, Crossref.

Mukamel, R., Ekstrom, A.D., Kaplan, J., Iacoboni, M. \& Fried, I. (2010). Single-neuron responses in humans during execution and observation of actions, in "Current Biology", 20 (8), p. 750-756, Crossref.

Press, C., Weiskopf, N. \& Kilner, J.M. (2012). Dissociable roles of human inferior frontal gyrus during action execution and observation, in "Neuroimage", 60 (3), p. 1671-1677, Crossref.

Rizzolatti, G., Fadiga, L., Gallese, V., Fogassi, L. (1996). Premotor cortex and the recognition of motor actions, in "Cognitive Brain Research", 3 (2), p. 131-141, Crossref.

Rizzolatti, G. \& Arbib, M. A. (1998). Language within our grasp, in “Trends in Neuroscience”, 21 (5), p. 188-194, Crossref.

Rizzolatti, G. \& Fogassi, L. (2014). The mirror mechanism: recent findings and perspectives, in "Philosophical Transactions of the Royal Society B", 369 (1644), Crossref.

Tallerman, M. (2007). Did our ancestors speak a holistic protolanguage?, in "Lingua", 117 (3), p. 579-604, Crossref.

Tomasello, M. (1995). Language Is Not an Instinct. Book review: S. Pinker, The Language Instinct: How the Mind Creates Language, New York, William Morrow, 1994, in “Cognitive Development”, 10 (1), p. 131-156, Crossref.

Tomasello, M., Kruger, A., \& Ratner, H. (1993). Cultural Learning, in "Behavioral and Brain Sciences”, 16 (3), p. 495-552, Crossref.

Wray, A. (1998). Protolanguage as a holistic system for social interaction, in “Language and Communication", 18 (1), p. 47-67, Crossref.

Wray, A. (2002). The Transition to Language, Oxford University Press. 